\title{
Projection of compact fractal sets: application to diffusion-limited and cluster-cluster aggregates
}

\author{
F. Maggi \\ Berkeley Water Center, Civil and Environmental Engineering, 413 O'Brien Hall, University of California, Berkeley, CA \\ 94720-1710, USA
}

Received: 4 March 2008 - Revised: 14 July 2008 - Accepted: 25 July 2008 - Published: 26 August 2008

\begin{abstract}
The need to assess the three-dimensional fractal dimension of fractal aggregates from the fractal dimension of two-dimensional projections is very frequent in geophysics, soil, and atmospheric sciences. However, a generally valid approach to relate the two- and three-dimensional fractal dimensions is missing, thus questioning the accuracy of the method used until now in practical applications. A mathematical approach developed for application to suspended aggregates made of cohesive sediment is investigated and applied here more generally to Diffusion-Limited Aggregates (DLA) and Cluster-Cluster Aggregates (CCA), showing higher accuracy in determining the three-dimensional fractal dimension compared to the method currently used.
\end{abstract}

\section{Introduction}

Fractal aggregates occur very commonly at many scales in geophysical observations such as, for instance, in cosmology (e.g., galaxy distribution and patterns at large scales, Coleman and Pietronero, 1992; Thieberger and Spiegel, 2000), physics of the atmosphere (e.g., clouds, aerosols, contaminant dusts, Friedlander, 1977; Shaw, 2003), soil sciences (e.g., soil water retention properties, Perrier et al., 1996), and sedimentology (e.g., flocculation of cohesive sediment, Kranenburg, 1994; Winterwerp, 1999; Maggi, 2007). Determining the three-dimensional (3-D) capacity dimension of geophysical fractal aggregates is particularly beneficial to scale geometrical properties (e.g., effective density, porosity, strength, mass density distribution, etc.) with a characteristic length scale (Meakin, 1991). However, direct assessment of the 3-D capacity dimension is rare in practice due to technical limitations of observational instruments. More likely, 2-D projections can be assessed from optical measurements. The
2-D capacity dimension has been used up to now to estimate the 3-D capacity dimension as follows. Given a 3-D fractal set (aggregate) $S_{3} \subseteq \mathbb{R}^{3}$ and its 2-D projection $S_{2} \subseteq \mathbb{R}^{2}$ obtained by application of the linear transformation (projection) $\mathcal{P}: \mathbb{R}^{3} \rightarrow \mathbb{R}^{2}$ the capacity dimensions $d_{0}$ of $S_{2}$ and $S_{3}$ are assumed to relate as $d_{0}\left(S_{2}\right)=\min \left\{2, d_{0}\left(S_{3}\right)\right\}$ (Vicsek, 1992; Meakin, 1998).

The inverse form

$d_{0}\left(S_{3}\right)= \begin{cases}d_{0}\left(S_{2}\right) & \text { for } d_{0}\left(S_{2}\right)<2, \\ \text { undetermined } \in[2,3] & \text { for } d_{0}\left(S_{2}\right)=2 .\end{cases}$

is commonly used to estimate $d_{0}\left(S_{3}\right)$ from $d_{0}\left(S_{2}\right)$. However, there are three major limitations using Eq. (1): (i) it assumes that $S_{2}$ and $S_{3}$ are indefinitely extended sets; (ii) for $d_{0}\left(S_{2}\right)=2$ the 3-D capacity dimension $d_{0}\left(S_{3}\right)$ is undetermined; (iii) for projections $\mathcal{P}: \mathbb{R}^{n} \rightarrow \mathbb{R}^{m}$ with $n>m$, the generalized dimensionality $d_{q}$ (Grassberger and Procaccia, 1983 ) is dimension-preserving only for $1<q \leq 2$, whereas for the capacity dimension $d_{0}$, which equals $d_{q}$ for $q=0$, the transformation $\mathcal{P}$ is not dimension-preserving (Hunt and Kaloshin, 1997; Thieberger and Spiegel, 2000).

These aspects rather suggest that, for compact (finite and closed) sets like the ones obtained by empirical observation, $d_{0}\left(S_{3}\right) \neq d_{0}\left(S_{2}\right)$ also when $d_{0}\left(S_{2}\right)<2$. This analysis questions the applicability of Eq. (1) to geophysical observations. Errors introduced using Eq. (1) were shown in Maggi and Winterwerp (2004) when applied to kaolinite aggregates formed in water suspensions. In the same instance, an empirical relation that used the perimeter-based fractal dimension $d_{P}$ was proposed to compute $d_{0}\left(S_{3}\right)$ from projections $S_{2}$, thus circumventing the use of the 2-D capacity dimension $d_{0}\left(S_{2}\right)$ in favor of alternative information not belonging to the generalized dimensionality $d_{q}$. This approach yielded the following (Maggi and Winterwerp, 2004)

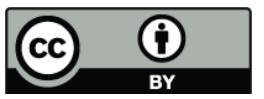

Correspondence to: F. Maggi

(fmaggi@berkeley.edu)

Published by Copernicus Publications on behalf of the European Geosciences Union and the American Geophysical Union. 


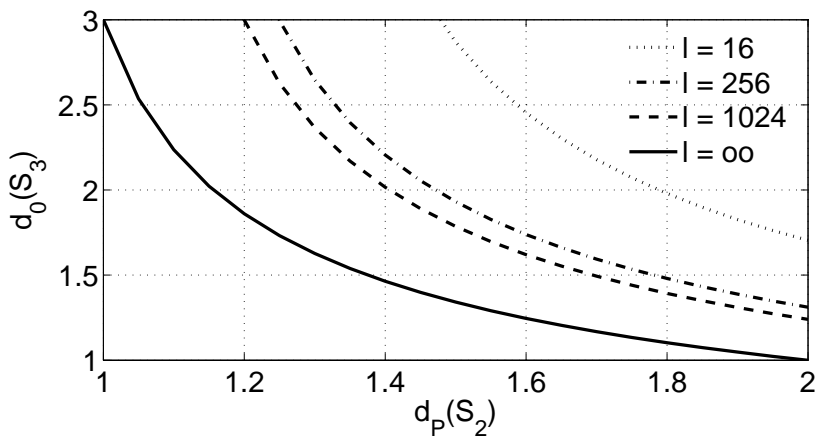

Fig. 1. Representation of the 3-D capacity dimension $d_{0}\left(S_{3}\right)$ as a function of the perimeter-based fractal dimension $d_{p}\left(S_{2}\right)$, Eq. (2), for different resolutions $\ell=L / \epsilon$, where $L$ is the length scale of the aggregate and $\epsilon$ is the box size of the $\epsilon$-covering.

$d_{0}\left(S_{3}\right)=\left[\frac{a(\ell)}{d_{P}\left(S_{2}\right)-b(\ell)}\right]^{1 / 2}$,

where $d_{p}=2 \log [P] / \log [A]$ is the perimeter-based fractal dimension (Meakin, 1998), with $P$ and $A$ the dimensionless perimeter and area of $S_{2}$, respectively, and $a(\ell)$ and $b(\ell)$ defined as

$b(\ell)=\frac{2[k(\ell)]^{2}-9 z(\ell)}{[k(\ell)]^{2}-9}, \quad a(\ell)=9[z(\ell)-b(\ell)]$,

with

$z(\ell)=\frac{\log [4 \ell-4]}{\log [\ell]}, \quad k(\ell)=z(\ell)[z(\ell)-1]+1$,

known for any given resolution $\ell=L / \epsilon$, with $L$ the size of $S_{2}$ and $S_{3}$, and $\epsilon$ the box size of the $\epsilon$-covering of $S_{2}$ (Maggi and Winterwerp, 2004). Equation (2) is not resolution-limited as far as the disequality $(4 \ell-4) \leq \ell^{\left(1+\sqrt{4 d_{0}\left(S_{3}\right)-3}\right) / 2}$ is satisfied (Maggi and Winterwerp, 2004). A representation of Eq. (2) is given in Fig. 1 for various values of $\ell$, showing that the accuracy in estimating $d_{0}\left(S_{3}\right)$ from $d_{P}\left(S_{2}\right)$ increases when $d_{P}\left(S_{2}\right)$ can range over the full interval $[1,2]$, that is, for increasing $\ell$.

The aim of this work is threefold. First, we derive the condition that any relationship between $d_{0}\left(S_{2}\right)$ and $d_{0}\left(S_{3}\right)$ must satisfy for compact fractal sets. Second, we show that both Eqs. (1) and (2) satisfy such a condition. Third, we show that Eq. (2) produces better results than Eq. (1), and that its validity extends to DLA (diffusion-limited) and CCA (clustercluster) aggregates, typologies which occur commonly in a variety of situations in geophysics.

\section{Method}

\subsection{Relationship between $d_{0}\left(S_{2}\right)$ and $d_{0}\left(S_{3}\right)$}

A general relationship between $d_{0}\left(S_{2}\right)$ and $d_{0}\left(S_{3}\right)$ can be derived as follows. For a compact fractal aggregate $S_{3} \subset \mathbb{R}^{3}$ of length scale $L$, the volume, $V$, scales as $V=L^{d_{0}\left(S_{3}\right)}$ with $0 \leq d_{0}\left(S_{3}\right) \leq 3$ the 3-D capacity dimension of $S_{3}$ (Meakin, 1991). In analogy, the projected area, $A$, scales as $A=L^{d_{0}\left(S_{2}\right)}$ with $0 \leq d_{0}\left(S_{2}\right) \leq 2$ the 2-D capacity dimension of the projected set $S_{2}$ of $S_{3}$. Under the assumption that $L$ is the length scale for both $S_{2}$ and $S_{3}$, the volume $V$ must be

$V \leq A \cdot L$

Substituting the scalings for $V$ and $A$ into Eq. (3) we obtain $L^{d_{0}\left(S_{3}\right)} \leq L^{d_{0}\left(S_{2}\right)+1}$, which implies

$d_{0}\left(S_{3}\right) \leq d_{0}\left(S_{2}\right)+1$.

This inequality sets the condition that must be respected whatever the function relating $d_{0}\left(S_{2}\right)$ and $d_{0}\left(S_{3}\right)$. Condition (4) can be generalized for projections $\mathcal{P}: \mathbb{R}^{n} \rightarrow \mathbb{R}^{m}$ of compact fractal sets in any embedding dimensions $n>m$ as

$d_{0}\left(S_{n}\right) \leq d_{0}\left(S_{m}\right)+(n-m)$.

Note that $d_{0}\left(S_{n}\right)=d_{0}\left(S_{m}\right)+(n-m)$ represents the relationship between $d_{0}\left(S_{n}\right)$ and $d_{0}\left(S_{m}\right)$ of an aggregate in $\mathbb{R}^{n}$ and its cross-section in $\mathbb{R}^{m}$ (Vicsek, 1992).

\subsection{Validation of Eq. (1) to relate $d_{0}\left(S_{2}\right)$ and $d_{0}\left(S_{3}\right)$}

Upon substitution of Eq. (1) into condition (4) for $d_{0}\left(S_{2}\right)<2$ we obtain

$d_{0}\left(S_{3}\right)=d_{0}\left(S_{2}\right) \leq d_{0}\left(S_{2}\right)+1$,

which always satisfies the disequality. In analogy, substituting Eq. (1) for $d_{0}\left(S_{2}\right)=2$ into condition (4) we obtain

$d_{0}\left(S_{3}\right) \in[2,3] \leq d_{0}\left(S_{2}\right)+1=3$,

which always satisfies the disequality.

\subsection{Validation of Eq. (2) to relate $d_{P}\left(S_{2}\right)$ and $d_{0}\left(S_{3}\right)$}

Assuming that disequality $(4 \ell-4) \leq \ell^{\left(1+\sqrt{4 d_{0}\left(S_{3}\right)-3}\right) / 2}$ is always satisfied (see Sect. 1), and for the case of infinite resolution $\ell \rightarrow \infty$ (Fig. 1), the functions $a(\infty)$ and $b(\infty)$ in Eq. (2) become $a=9 / 8$ and $b=7 / 8$ (Maggi and Winterwerp, 2004). By introducing Eq. (2) into condition (4) we obtain

$$
\begin{aligned}
d_{0}\left(S_{3}\right)= & {\left[\frac{9 / 8}{d_{P}\left(S_{2}\right)-7 / 8}\right]^{1 / 2} \leq d_{0}\left(S_{2}\right)+1, } \\
& \frac{9 / 8}{\left[d_{0}\left(S_{2}\right)+1\right]^{2}}+7 / 8 \leq d_{P}\left(S_{2}\right),
\end{aligned}
$$




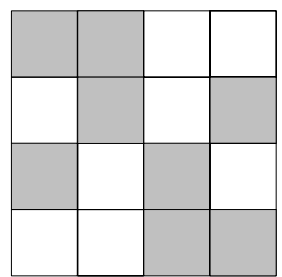

Set B

$$
\begin{aligned}
d_{0} & =\frac{\log [8]}{\log [4]}=1.5 \\
d_{P} & =2 \frac{\log [8]}{\log [8]}=2
\end{aligned}
$$

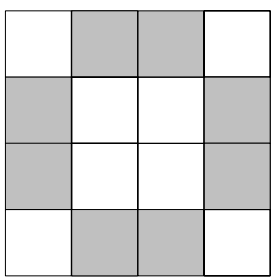

Set C

$$
\begin{array}{r}
d_{0}=\frac{\log [8]}{\log [4]}=1.5 \\
d_{P}=2 \frac{\log [8]}{\log [12]}=1.67
\end{array}
$$

Fig. 2. Examples of sets with identical capacity dimension $d_{0}$ and different perimeter segmentation. Computation of the perimeterbased fractal dimension $d_{P}$ has been done neglecting the inner voids, according to Maggi and Winterwerp (2004).

that can be analyzed analytically for the two extreme cases of massive and linelike projections. For fully massive projections (i.e., $d_{0}\left(S_{2}\right)=2$ and $d_{P}\left(S_{2}\right)=1$, Maggi and Winterwerp, 2004), Eq. (6) becomes

$$
\frac{9 / 8}{(2+1)^{2}}+7 / 8=1 \leq 1,
$$

which satisfies the disequality. Analogously, for linelike projections (i.e., $d_{0}\left(S_{2}\right)=1$ and $d_{P}\left(S_{2}\right)=2$ ), and Eq. (6) becomes

$$
\frac{9 / 8}{(1+1)^{2}}+7 / 8=\frac{37}{32} \leq 2 \text {, }
$$

which satisfies the disequality.

While Eq. (2) satisfies condition (4) for massive and linelike projections, it is not known whether this holds also for projected irregular sets with $d_{0}\left(S_{2}\right)$ and $d_{P}\left(S_{2}\right)$ in the range $(1,2)$. To this end, we test Eq. (2) to compute $d_{0}\left(S_{3}\right)$ of aggregates of different nature and geometrical properties, and we compare the results with those obtained using Eq. (1) with the aim to assess which approach enables better reconstructions of $d_{0}\left(S_{3}\right)$ from $S_{2}$.

\section{Results}

3.1 Application to diffusion-limited and cluster-cluster aggregates

Geophysical fractal aggregates produced by various kinematic processes generally show different geometrical organization. For instance, DLA aggregates, which are formed by attachment of individual particles that move in space by diffusion, are featured by radial development of stellar-like branchings. Instead, CCA aggregates, which are formed by clusters of particles that recursively attach to each other and move in space by diffusion, display clustered masses connected by bridges, forming (sometimes closed) rings. Fully

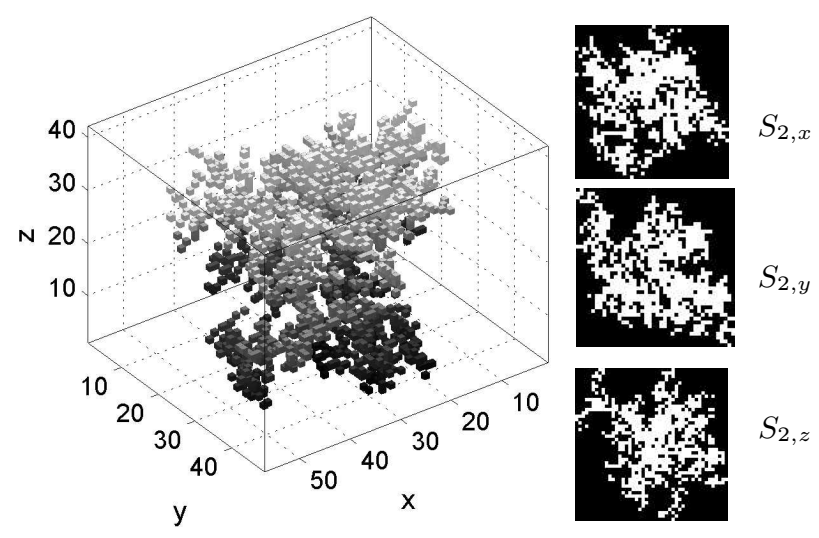

(a) DLA

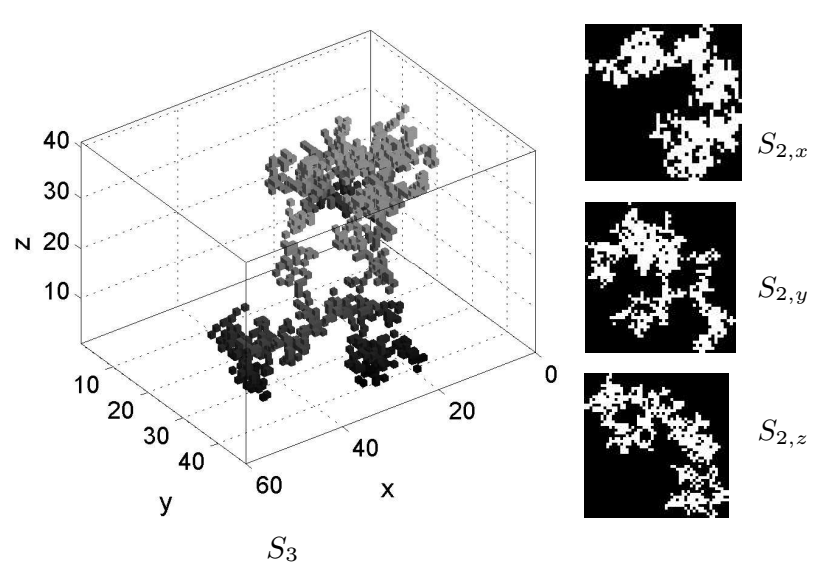

(b) $\mathrm{CCA}$

Fig. 3. Examples of DLA and CCA aggregates. Their 2-D projections are shown beside the 3-D views.

developed DLA and CCA aggregates usually have different capacity dimensions in $\mathbb{R}^{3}$, that is $d_{0}(\mathrm{DLA}) \approx 2.5$ and $d_{0}(\mathrm{CCA}) \approx 1.8$, respectively (Vicsek, 1992). However, projections $\mathcal{P}$ of those aggregates may have similar capacity dimensions $d_{0}\left(S_{2}\right)$ in $\mathbb{R}^{2}$, thus preventing to discerning aggregate type and 3-D capacity dimension from the capacity of $S_{2}$. A conceptual example is given in Fig. 2, where two sets $B$ and $C$ of different outlook (ramified the former and ringshaped the latter) have identical capacity dimension $d_{0}$ but different perimeter-based fractal dimension $d_{P}$ in $\mathbb{R}^{2}$.

For this test, we have constructed 10 DLA aggregates and 10 CCA aggregates of 1000 seeds according to the algorithms described in Vicsek (1992) and Meakin (1998) (Fig. 3). Next, 2-D projections in the three principal directions have been computed. From these we have calculated the average $\overline{d_{P}\left(S_{2}\right)}$ and $\overline{d_{0}\left(S_{2}\right)}$, terms used in Eqs. (1) and (2) to compute $d_{0}\left(S_{3}\right)$. When Eq. (2) has been used, an additional computation has been carried out to make sure that the disequality $(4 \ell-4) \leq \ell^{\left(1+\sqrt{4 d_{0}\left(S_{3}\right)-3}\right) / 2}$ was satisfied (data not shown). The values of $d_{0}\left(S_{3}\right)$ so computed have 

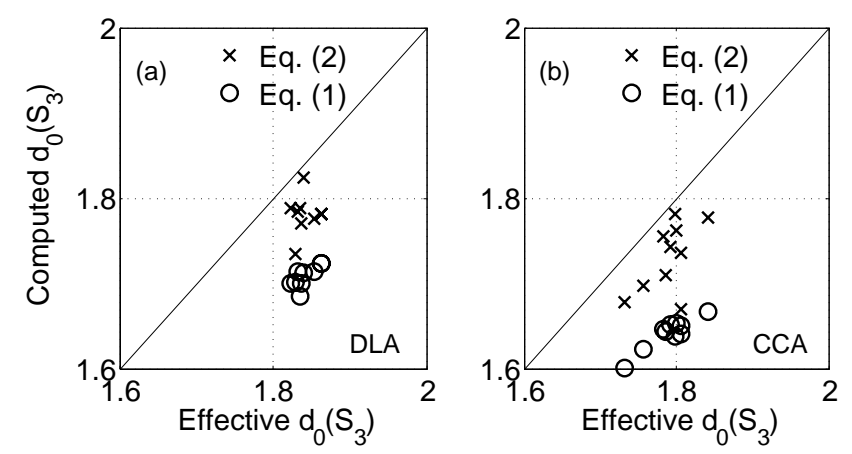

Fig. 4. Comparison between the effective 3-D capacity dimension $d_{0}\left(S_{3}\right)$ obtained by construction and the one calculated from Eq. (1) and Eq. (2) for (a) DLA aggregates and (b) CCA aggregates.

been compared to the ones obtained by construction (effective $d_{0}\left(S_{3}\right)$ in Fig. 4).

The results in Fig. 4 show that application of Eq. (2) always gives better estimations of $d_{0}\left(S_{3}\right)$ from the projections compared to Eq. (1). The loss of information induced by $\mathcal{P}$ on the sets affects their capacity, but preserves the information at the boundary of the projections. Because the perimeter-based fractal dimension $d_{P}\left(S_{2}\right)$ is representative of the boundary segmentation rather than the set capacity, Eq. (2) allows better prediction of $d_{0}\left(S_{3}\right)$. These results show that the geometrical organization of the boundary of the sets $S_{3}$ can be considered $\mathcal{P}$-invariant, and that this aspect can be fruitfully exploited to estimate $d_{0}\left(S_{3}\right)$ of real geophysical fractal aggregates.

\subsection{Exceptions}

Two particular cases exist for which Eq. (2) is biased: regular solids with porous structure (sponge-like) and Euclidean solids with fractal surface (rock-like).

For the former (sponge), consider as hypothesis a regular cube with a high level of fine porosity so that $d_{0}\left(S_{3}\right)<3$ (Fig. 5a). The projected image $S_{2}$ appears fully massive and its contour does not present segmentation. Consequently, $d_{P}\left(S_{2}\right)=1$ for $\ell \rightarrow \infty$, and from Eq. (2) we obtain $d_{0}\left(S_{3}\right)=3$, which contradicts the hypothesis.

For the latter (rock), consider as hypothesis a fully massive solid with capacity dimension $d_{0}\left(S_{3}\right)=3$ and with a fractal (rough and irregular) surface (Fig. 5b). Its projected image $S_{2}$ is fully massive but the contour shows a high level of perimeter segmentation. As a consequence, $d_{P}\left(S_{2}\right)>1$ and from Eq. (2) we obtain $d_{0}\left(S_{3}\right)<3$, which again contradicts the hypothesis.
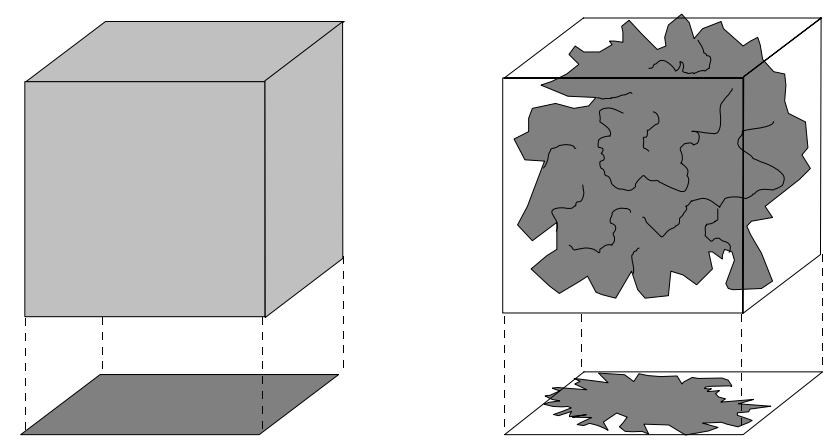

Fig. 5. (left) regular solid with porous (fractal) structure and massive (non-fractal) projection and, (right), Euclidean solid with fractal surface and massive projection with highly segmented contour. Light gray means porous structure, while dark gray means Euclidean structure.

\section{Summary}

A simple method to compute the three-dimensional capacity dimension from two-dimensional projections of compact fractal sets is tested on DLA and CCA aggregates, and compared to the approach more commonly used in geophysics. The method presented here makes use of the perimeter-based fractal dimension and does not obey the mathematical limitations that apply to the capacity dimension and the generalized dimensionality. The numerical simulations presented here show that this method produces better predictions of the three-dimensional capacity dimension in the two aggregate typologies (DLA and CCA) analyzed here compared to the current state-of-the-art method.

Acknowledgements. The author is particularly grateful to William Riley for his comments on the contents and presentation of this work.

Edited by: U. Feudel

Reviewed by: two anonymous referees

\section{References}

Coleman P. and Pietronero L.: The fractal structure of the Universe, Phys. Rep. 213, 311-389, 1992.

Friedlander S. K.: Smoke, Dust and Haze, fundamentals of aerosol behavior. Wiler, New York, 1977.

Grassberger H. G. E. and Procaccia I.: Characterization of strange attractors, Phys. Rev. Lett. 50(5), 346-349, 1983.

Hunt B. R. and Kaloshin V. Y.: How projections affect the dimension spectrum of fractal measures, Nonlinearity 10, 1031-1046, 1997.

Kranenburg C.: On the fractal structure of cohesive sediment aggregates, Continental and Shelf Science, 39, 451-460, 1994.

Maggi F. and Winterwerp J. C.: Method for computing the three-dimensional capacity dimension from two-dimensional 
projections of fractal aggregates, Phys. Rev. E, 69, 011405, doi:10.1103/PhysRevE.69.011405, 2004.

Maggi F.: Variable fractal dimension: a major control for floc structure and flocculation kinematics of suspended cohesive sediment, J. Geophys. Res., 112, C07012, doi:10.1029/2006JC003951, 2007.

Meakin P.: Fractal aggregates in geophysics, Reviews of Geophysics, 29, 317-354, 1991.

Meakin P.: Fractals, Scaling and Growth far from Equilibrium, Cambridge University Press, Cambridge, England, 1998.
Perrier E., Rieu M., Sposito G., and de Marsily G.: Models of the water retention curve for soils with a fractal pore size distribution, Wat. Res. Res., 32(10), 3025-3031, 2002.

Shaw R. A.: Particle-turbulence interactions in atmospheric clouds, Annu. Rev. Fluid Mech. 35, 183-227, 2003.

Thieberger R. and Spiegel E. A.: Luminosity effects in projected fractals, Bull. of Astron. Soc. India 30, 461, 2000.

Vicsek T.: Fractal growth phenomena, World Scientific, Singapore, 1992.

Winterwerp J. C.: On the dynamics of high-concentrateed mud suspensions, Ph.D. Thesis, Delft University of Technology, 1999. 